University of Massachusetts Amherst

ScholarWorks@UMass Amherst

Chemistry Department Faculty Publication Series

Chemistry

2012

\title{
Improving the accuracy and precision of an arsenic field test kit: increased reaction time and digital image analysis
}

James Kearns

University of Massachusetts Amherst

Julian Tyson

University of Massachusetts Amherst

Follow this and additional works at: https://scholarworks.umass.edu/chem_faculty_pubs

Part of the Chemistry Commons

\section{Recommended Citation}

Kearns, James and Tyson, Julian, "Improving the accuracy and precision of an arsenic field test kit: increased reaction time and digital image analysis" (2012). Analytical Methods. 1354.

Retrieved from https://scholarworks.umass.edu/chem_faculty_pubs/1354 


\title{
Improving the accuracy and precision of an arsenic field test kit: increased reaction time and digital image analysis
}

\author{
James Kearns and Julian Tyson* \\ Received 9th October 2011, Accepted 10th April 2012 \\ DOI: $10.1039 / \mathrm{c} 2 \mathrm{ay} 05655 \mathrm{k}$
}

Two procedures to improve the performance of the Hach EZ test kit for quantifying inorganic arsenic concentrations in drinking water have been investigated. In the first, a digital image of the colored spot formed on the test strip, obtained with a flat-bed scanner was analyzed, by the computer program Colors, for the R, G, and B values. Calibrations were constructed by plotting the B values as a function of concentration. Agreement between the experimentally determined B-values and those of the printed chart was only obtained by either increasing the reaction time (to $40 \mathrm{~min}$ ) or increasing the reaction temperature. The precision as a function of concentration was quantified. A comparison with previously estimated values for visual comparison of the colours, showed that the improved precision of the digital analysis would produce fewer false positive and fewer false negative results at the important threshold values of 10 and $50 \mu \mathrm{g} \mathrm{L}^{-1}$. By running the test for $24 \mathrm{~h}$, improved performance at the low concentration (around $10 \mu \mathrm{g} \mathrm{L}^{-1}$ ) end of the response scale was obtained.

\section{Introduction}

Arsenic compounds, which are widely distributed in the environment as a consequence of natural processes and anthropogenic activities, are implicated in the adverse health of millions of people around the world. ${ }^{1}$ The majority of those exposed are drinking contaminated water. ${ }^{2}$ Chronic consumption of arseniccontaminated water causes skin lesions, neurological disorders and cancers, including cancer of the kidneys and lungs. ${ }^{3}$ Probably the greatest suffering is in Bangladesh and West Bengal, India, whose rural communities are currently battling "the largest mass poisoning of a population in history." 4 The World Health Organization currently suggests a limit of $10 \mu \mathrm{g} \mathrm{L}^{-1}$ for arsenic in water, which is also the "maximum contaminant level" set by the US Environmental Protection Agency; however, $50 \mu \mathrm{g}$ $\mathrm{L}^{-1}$ is currently the threshold value in Bangladesh and India. ${ }^{5}$

In the rural regions of southeast Asia, and elsewhere, affected populations often obtain their water from tube wells sunk into shallow arsenic-contaminated aquifers. Such locations are generally remote from laboratory-based facilities, making laboratory analysis of the very large numbers of local well waters impractical. ${ }^{6}$ In 2005, Melamed reviewed technologies with field measurement potential for monitoring arsenic in the environment. ${ }^{7}$ He concluded: "Accurate, fast measurement of arsenic in the field remains a technical challenge... the central goal of developing field assays that reliably and reproducibly quantify arsenic has not been achieved." Bangladesh alone has more than ten million tube wells, and field test-kits are the only realistic

Department of Chemistry, University of Massachusetts Amherst, MA 01003,USA.E-mail: tyson@chem.umass.edu means of measuring the arsenic content of the water abstracted. ${ }^{6}$ The analysis is based on the Gutzeit modification of the Marsh reaction, in which arsine gas $\left(\mathrm{AsH}_{3}\right)$, formed by reaction of inorganic arsenate or arsenite with zinc in acid solution, reacts with mercuric bromide, impregnated into a paper strip exposed to the head-space of the reaction vessel, to produce a yellowbrown product. The colour is related to the concentration of arsenic in solution, which is found by comparing the colour of the strip with colours on a printed chart provided by the manufacturer. There has been adverse criticism voiced over the performance of earlier versions of these field test kits. Hossain ${ }^{8}$ alludes to the Bangladesh water crisis and the practice of painting a Bangladeshi tube well green if the water contains less than $50 \mu \mathrm{g} \mathrm{L}^{-1}$ and red if it contains more than $50 \mu \mathrm{g} \mathrm{L}^{-1}$, when he writes (in 2006) "field kits used to measure As in the region's groundwater are unreliable" and "many wells in Bangladesh have been labeled incorrectly." In an earlier study, published in 2002, Rahman et al. ${ }^{9}$ conclude, after evaluating results from the kits made by Merck, the National Institute of Preventional and Social Medicine (NIPSOM) in India, the Asia Arsenic Network (AAN), the All India Institute of Hygeine and Public Health (AII\&PH), and Hach, that "millions of dollars are being spent without scientific validation of the field kit method."

However, the performances of more recent versions of the kits are much improved. Van Geen et al. compared ${ }^{10}$ results obtained with the Hach EZ kit with those based on laboratory measurements by HG-AAS for the analysis of the water from 799 Bangladeshi tube wells. They found that provided the reaction time was doubled to $40 \mathrm{~min}$, the field kit results were accurate (with respect to the $50 \mu \mathrm{g} \mathrm{L}^{-1}$ value) for $88 \%$ of the samples. Steinmaus et al. evaluated ${ }^{11}$ the Hach EZ kit (and the Quick Arsenic kit) in 
the context of the $10 \mu \mathrm{g} \mathrm{L}^{-1}$ standard by the analysis of 136 water samples from western Nevada, USA. The laboratory reference method involved HG-AFS. They increased the reaction time to $40 \mathrm{~min}$ for the Hach kit as suggested by van Geen et al. and found that for the 109 samples that contained more than $15 \mu \mathrm{g} \mathrm{L}^{-1}$, the EZ kit correctly identified the concentration as being above $10 \mu \mathrm{g} \mathrm{L}^{-1}$. For the 27 samples that contained less than $10 \mu \mathrm{g} \mathrm{L}^{-1}$, the Hach EZ kit registered 2 false positives. The status of test kits based on the Gutzeit reaction has been reviewed by Tyson. ${ }^{12}$

The US Environmental Protection Agency's Environmental Technology Verification (ETV) Program ${ }^{13}$ has, for several years, been evaluating arsenic test kits according to a rigorous and extensive set of standard protocols. The ETV program has, to date, evaluated eight test kits based on the Gutzeit reaction submitted by the manufacturers. On testing these kits for accuracy when measuring $10 \mu \mathrm{g} \mathrm{L}^{-1}$ As, the percentage of false positives was as high as 18 and the percentage of false negatives was as high as 62 .

The issue of the occurrence of false positives and false negatives was examined in detail by Kinniburgh and Kosmus. ${ }^{6}$ They pointed out that the frequency of these was related to the precision of the measurement, which would be a function of the analyte concentration. To calculate this, they adopted the model of Thompson and Howarth, ${ }^{14} s_{\mathrm{c}}=s_{0}+k C$ where $s_{\mathrm{c}}$ is the standard deviation of replicate analyses at concentration $C, s_{0}$ is the standard deviation at zero concentration, and $k$ is a constant. For the Hach EZ kit, they calculated, based on data supplied by the Hach company, $s_{0}$ to be $7 \mu \mathrm{g} \mathrm{L}^{-1}$ and $k$ to be 0.3 . They plotted the percentage chance of an inaccurate result as a function of analyte concentration for a $50 \mu \mathrm{g} \mathrm{L}^{-1}$ decision value. This plot showed, for example, that for a field test-kit, the probability of a false negative for a sample of $100 \mu \mathrm{g} \mathrm{L}^{-1}$ would still be about $5 \%$, and the probability of a false positive at $25 \mu \mathrm{g} \mathrm{L}^{-1}$ would be about $2 \%$. They applied their treatment to 3208 real samples. In a simulation of 1000 analyses of each, they deduced that the Hach EZ kit would misclassify $12 \%$ of the wells, whereas a laboratory-based instrumental method (for which $s_{0}=0.3 \mu \mathrm{g} \mathrm{L}^{-1}$ and $k=0.088$ ) would misclassify just under $1 \%$ of the wells. Clearly if the precision of the test kit could be improved, the reliability of the results would be improved (i.e. numbers of wells misclassified would be decreased).

We propose that one possible way of improving the precision of colorimetric determinations is to work with digital images obtained with a flat-bed scanner, which was first described in the 1993 paper by Durst and co-workers. ${ }^{15,16}$ They pointed out that the grayscale value was independent of the absorption spectrum of the dye used. The grayscale approach was also used by Bannur et al. ${ }^{17}$ and by Johnson. ${ }^{18}$ Abrazheev et al. ${ }^{19}$ determined arsenic by a modification of the Gutzeit method in which a function analogous to absorbance was calculated. The first report of the use of the separated red, green, and blue (RGB) colour intensities was in 2002 by Kompany-Zareh et al. ${ }^{20}$ who derived a similar absorbance function. Paciornik et al. ${ }^{21}$ compared the performance of the RGB; cyan, magenta, yellow (CMY); and hue, lightness, saturation (HLS) colour space models and concluded that the best parameter to use was the hue, H. More recently, Sharma et al. ${ }^{22}$ analyzed the images produced by reaction of arsenic with sulfanilic acid and $N$-(1-naphthyl)ethylene diamine dihydrochloride solution. The detection limit of $60 \mu \mathrm{g} \mathrm{L}^{-1}$ is, unfortunately, not low enough to be of any use for the monitoring of arsenic in groundwater. Mathews et al. ${ }^{23}$ analyzed JPEG images by a program, Colors, that is available via the supplemental material on the journal website. ${ }^{24}$ While the current manuscript was under review, Salman et al. described a method for arsenic based on the Gutzeit method in which the colored spots were scanned and a colour density value computed by adding the $\mathrm{R}, \mathrm{G}$, and $\mathrm{B}$ values together. ${ }^{25}$

In this paper, we present results of studies to improve measurement precision by the analysis of the digital images, obtained with a flat-bed scanner, of the exposed test-strips from the Hach EZ test kit for the determination of arsenic in solution. We also present results of our studies of the effects of temperature and time. We discuss the implications of these findings for the analytical performance of such tests.

\section{Experimental}

\section{Test kit vessels}

Reaction vessels and lids were obtained from the Hach Company (Loveland, CO) in the EZ Arsenic Test Kit (cat. 2822800).

\section{Reagents and standards}

High purity water $(18 \mathrm{M} \Omega \mathrm{cm})$ was obtained from a Barnstead/ Thermodyne (Dubuque, IA) E-pure unit. The reagents used for arsine gas generation were those provided with the EZ Arsenic Test Kit, namely sulfamic acid (cat. 28229-99) and zinc (cat. 28230-99). Standard solutions were prepared from laboratory grade sodium arsenite, $\mathrm{NaAsO}_{2}$, cat. 225I) from Fisher Scientific (Pittsburgh, PA). Standard solutions of arsenic were prepared in deionized water with the concentrations ranging from $0,10,25$, 50, 100, 250, and $500 \mu \mathrm{g} \mathrm{L}{ }^{-1}$.

\section{Measurement procedure}

All experiments were carried out according to the procedure described by the manufacturer of the test kit. Reaction vessels were filled with $50 \mathrm{~mL}$ of the given solution. Sulfamic acid was added and dissolved. The second reagent, zinc, was added, and the vessel was capped with a cap into which a test strip had been inserted and which contained a pea-sized piece of cotton wool in the holder on the inside face. The cotton wool was not moistened with the lead acetate solution provided (the procedure for the removal of any interference by sulfide) as only standard solutions were involved. During the measurement, a significant amount of hydrogen gas is evolved. When bubbles burst at the surface, aerosol droplets can be ejected that reach the mercuric bromide sensing surface of the strip, giving rise to uneven coloration. Except where indicated, five replicate measurements were made.

\section{Digital image analysis}

The strips were scanned with an Epson 2480 Perfection Photo flat-bed scanner operating at $600 \mathrm{dpi}$ and a 24-bit colour scale. The resulting images were cropped to display only the colored reaction product. Red, blue, and green intensity values were determined by Colors, run on a Windows XP platform, downloaded from the Journal of Chemical Education. ${ }^{24}$ The R, G, and 
B values for an image were each assigned a value from 0 to 255 . The colour intensity values were recorded in Microsoft Excel, which was used for calculations and curve-fitting.

The colored reference chart printed on the side of the strip container was scanned and the average red, green and blue pixel intensities for each spot determined. The chart, shown in Fig. 1, was reconstructed with Adobe Illustrator so that the spots have uniform colour.

\section{Method development}

Reaction time. The manufacturer currently recommends removing the strips after $20 \mathrm{~min}$. To investigate the effect of increasing the reaction time, a series of experiments was performed for each concentration in which the test strips were removed at 20, 30, $40 \mathrm{~min}$, and at $24 \mathrm{~h}$.

Temperature. The manufacturer does not specify a recommended operating temperature, and most reactions were run at room temperature $\left(20^{\circ} \mathrm{C}\right)$. To investigate the effect of operating temperatures typical of, say, Bangladesh, measurements were made at $35{ }^{\circ} \mathrm{C}$ for a reaction time of $20 \mathrm{~min}$. The reaction vessels were weighted and submerged so that the liquid levels in the vessels were below the water level in a thermostatically controlled water bath.

Data analysis. Plots of average blue pixel intensity, measured by the Colors program, as a function of arsenic concentration were constructed. To estimates the standard deviation in the concentration domain, $s_{\mathrm{c}}$, the quadratic equation corresponding to each successive group of three points, calculated in Excel, was solved for the concentration values that corresponded to the limits of \pm one standard deviation in the response domain (the blue intensity value). To examine the validity of the obtain Thompson-Howarth model and to obtain estimates of $k$ and $s_{0}$, plots of $s_{\mathrm{c}}$ as a function of $C$ were created and examined for the agreement with the straight line relationship described above.

\section{Results and discussion}

\section{Analysis of image of calibration chart}

The reference chart for the Hach EZ kit, in which the colours expected after $20 \mathrm{~min}$ reaction time for arsenic concentrations ranging from 0 to $500 \mu \mathrm{g} \mathrm{L}^{-1}$, is shown in Fig. 1. The difficulties of interpolating between adjacent colours can be seen, as can the difficulties of distinguishing between the responses of concentrations just below and above the $10 \mu \mathrm{g} \mathrm{L}^{-1}$ and $50 \mu \mathrm{g} \mathrm{L}^{-1}$ values.

The mean $\mathrm{R}, \mathrm{G}$ and $\mathrm{B}$ intensity values are shown in Table 1 , from which it can be seen that the $\mathrm{B}$ values are most responsive to the changes in the colours of the reaction spots on the test strips.

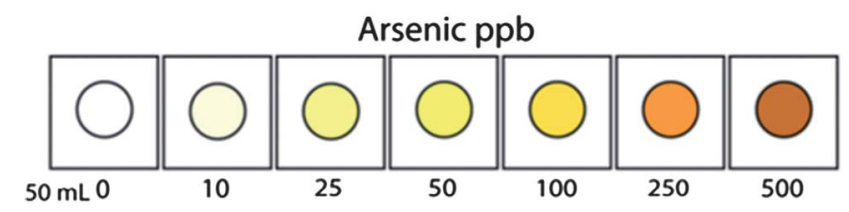

Fig. 1 Scanned image of the color chart corresponding to solutions containing $0,10,25,50,70,300$, and $500 \mu \mathrm{g} \mathrm{L}^{-1}$ of arsenic.
Table 1 Red, green and blue values from a scan of the Hach kit colour chart

\begin{tabular}{lllr}
\hline $\begin{array}{lll}\text { As(III) } \\
\text { concentration } \mu \mathrm{g} \mathrm{L}^{-1}\end{array}$ & Red & Green & Blue \\
\hline 0 & 255 & 255 & 255 \\
10 & 254 & 254 & 223 \\
25 & 254 & 254 & 137 \\
50 & 254 & 254 & 101 \\
100 & 252 & 222 & 65 \\
250 & 251 & 157 & 53 \\
500 & 201 & 113 & 44 \\
\hline
\end{tabular}

The primary trend is that the intensity of the reflected "blue" light (i.e. as defined by the blue filters in the scanner) decreased as concentration of arsenic increased.

\section{Effect of reaction time}

The mean blue intensity values for reaction times of 20,30, 40 min, and $24 \mathrm{~h}$ and for the Hach kit colour chart are shown in Table 2; the plots corresponding plots of blue intensity value as a function of concentration are shown in Fig. 2. It can be seen that curves have an approximately exponential shape, and that even for a 40 minute reaction time, the blue values are not as intense as the values in the printed colour chart. As the values for the 24 hour reaction time are more intense than those of the printed chart, it maybe concluded that the best match to the chart for reaction at room temperature would be obtained for reaction times longer than 40 minutes but shorter than 24 hours. We have preliminary results that indicate that this might be as long as $4 \mathrm{~h}$; however, the situation is complicated by the fact we also have evidence that the colour fades on prolonged exposure to the headspace vapours. Clearly reaction times of hours are not compatible with the activities of a single technician tasked with visiting as many sites as possible in the working day, but are less problematic if the tests are being performed by multiple individuals. We therefore endorse the suggestion by van Geen et al. ${ }^{10}$ that when operating at $20{ }^{\circ} \mathrm{C}$, the reaction time be increased to $40 \mathrm{~min}$. A $24 \mathrm{~h}$ reaction time can work well with the scheduling of classes in schools, where students can set up the test in one laboratory class period and "read" the strip during the class the following day. In addition, it can be seen that the slope of the calibration for the low concentrations (between 0 and about $10 \mu \mathrm{g} \mathrm{L}^{-1}$ ) is greatest for the $24 \mathrm{~h}$ version of the test, and thus more reliable results for the values near the WHO's critical value will be obtained by increasing the reaction time.

\section{Effect of temperature}

The blue intensities obtained after $20 \mathrm{~min}$ at $35^{\circ} \mathrm{C}$ are also shown in Table 2 and the corresponding plots of blue intensity as a function of concentration are shown in Fig. 2. It maybe seen that raising the reaction temperature has a marked effect on the outcome of the test and that the values obtained at $35^{\circ} \mathrm{C}$ are closer to the values for the printed chart than the values obtained at $20{ }^{\circ} \mathrm{C}$. Thus, it would seem appropriate for the manufacturers of such tests to include some commentary about the temperature range for which the printed chart is considered appropriate and 
Table 2 Mean blue values for reactions run for $20,30,40 \mathrm{~min}$ at $20^{\circ} \mathrm{C}$ and for $20 \mathrm{~min}$ at $35^{\circ} \mathrm{C}$

\begin{tabular}{llllrr}
\hline As(III) concentration $\mu \mathrm{g} \mathrm{L}^{-1}$ & Hach colour chart & $20 \mathrm{~min}$ & $30 \mathrm{~min}$ & $40 \mathrm{~min}$ & $24 \mathrm{~h}$ \\
\hline 0 & 255 & 255 & 255 & 255 & 255 \\
10 & 223 & 249 & 241 & 233 & 154 \\
25 & 137 & 240 & 210 & 162 & 128 \\
50 & 101 & 211 & 132 & 140 & 255 \\
100 & 65 & 187 & 118 & 106 & 110 \\
250 & 53 & 157 & 101 & 85 & 245 \\
500 & 44 & 124 & & 85 \\
\hline
\end{tabular}

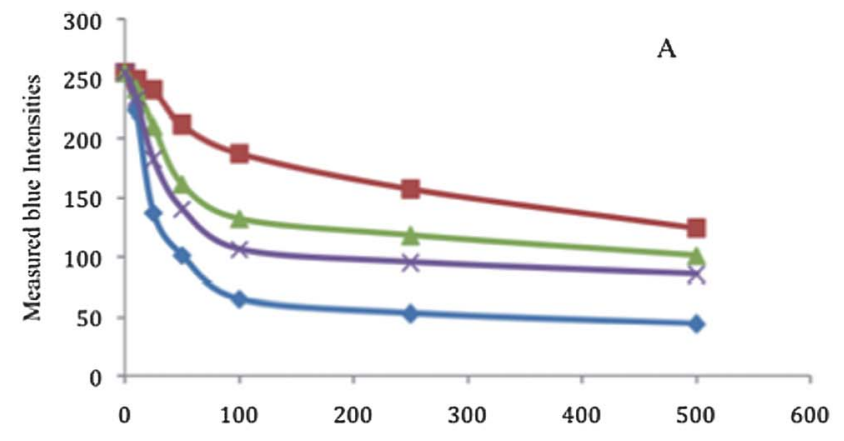

Concentration of As (III) in $\mu \mathrm{g} \mathrm{L}^{.1}$

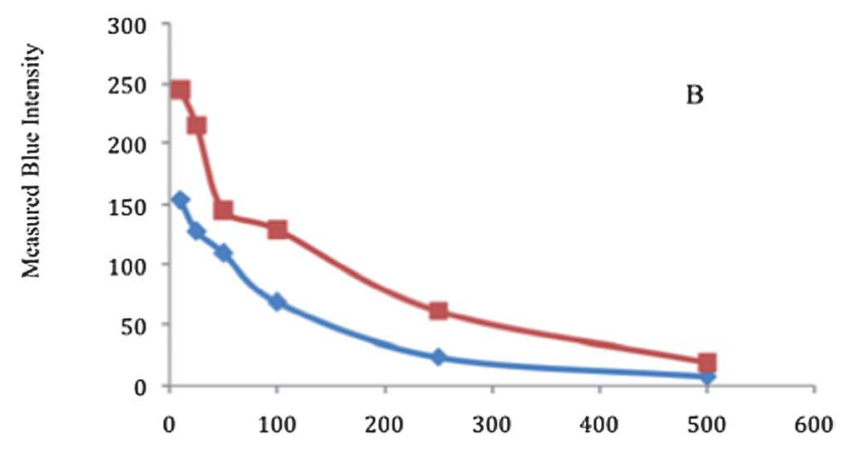

Concentration of As (III) in $\mu \mathrm{g} \mathrm{L}^{-1}$

Fig. 2 (A) A comparison of the measured blue intensities versus concentration of $\mathrm{As}(\mathrm{III})$ in $\mu \mathrm{g} \mathrm{L}^{-1}$ at $20 \mathrm{~min}$ (square), $30 \mathrm{~min}$ (triangle), 40 min (X) (all experiments at $20^{\circ} \mathrm{C}$ ) and the Hach color-chart (diamond). (B) A comparison of the measured blue intensities versus concentration of As(III) in $\mu \mathrm{g} \mathrm{L}^{-1}$ at $35{ }^{\circ} \mathrm{C}$ and $20 \mathrm{~min}$ (square) and $24 \mathrm{~h}$ and $20{ }^{\circ} \mathrm{C}$ (diamond).

maybe print more than one version of the chart corresponding to different temperatures. The same comment is relevant to the effect of reaction time. We suggest that it might be possible to create an algorithm that could be applied to correct for the effects of time and temperature and allow a more accurate match between the measured blue intensity and the intensities in the printed chart.

\section{Precision}

The standard deviations in blue intensity values $\left(s_{\mathrm{B}}\right)$ and the corresponding standard deviations in concentration $\left(s_{\mathrm{c}}\right)$ from values from scanned strips run at $20,30,40 \mathrm{~min}, 24 \mathrm{~h}$ at $20^{\circ} \mathrm{C}$ and for $20 \mathrm{~min}$ at $35^{\circ} \mathrm{C}$ are given in Table 3 . It maybe seen that the standard deviations in both domains increase as concentration increased as predicted by the Thompson and Howarth model. ${ }^{14}$ A plot of $s_{\mathrm{c}}$ as a function of $C$ for 20 min reaction at room temperature is shown in Fig. 3; while these data show reasonable agreement with the linear model, inspection of the data for other reaction condition shows that the relationships are not all linear. The nature of the response curves, especially those in Fig. 2A, is such that there are two fairly distinct response regions, below $100 \mu \mathrm{g} \mathrm{L}^{-1}$ and above $100 \mu \mathrm{g} \mathrm{L}^{-1}$. In the latter region, the slope of the response plot is much smaller than that in the former and so comparable variations ( \pm one standard deviation) in blue intensity would give rise to much larger variations in $C$. Better precision would probably be obtained for the determinations of concentrations above $100 \mu \mathrm{g} \mathrm{L}^{-1}$ if the green intensity values were used as the measure of concentration rather than the blue intensity values. However, as the most important consideration is whether the concentration in a ground water sample is above or below $50 \mu \mathrm{g} \mathrm{L}^{-1}$ or $10 \mu \mathrm{g} \mathrm{L}^{-1}$, depending on the part of the world in which the test is conducted, strategies for improving the precision at above $100 \mu \mathrm{g} \mathrm{L}^{-1}$ are less important than considerations of precision (and accuracy, discussed below) at lower concentrations. For most of the reaction conditions, the relationship between $s_{\mathrm{c}}$ and $C$ was better described by an exponential function than a linear function (results not shown).

The values of $s_{\mathrm{c}}$ estimated by Kinniburgh and Kosmus for the Hach test kit at $10 \mu \mathrm{g} \mathrm{L}^{-1}$ and $50 \mu \mathrm{g} \mathrm{L}^{-1}$ are $10 \mu \mathrm{g} \mathrm{L}^{-1}$ and $22 \mu \mathrm{g} \mathrm{\textrm {L } ^ { - 1 }}$, respectively. ${ }^{6}$ These values are given in Table 4, together with the values calculated from the standard deviations of 5 replicates of the responses to these two solutions at the various times and temperatures. The precisions at $30 \mathrm{~min}$, $40 \mathrm{~min}$ and $24 \mathrm{~h}$ were significantly smaller than the Kinniburgh and Kosmus values, based on a one-tailed $F$-test at the $95 \%$ confidence level. On this basis, the values for $20 \mathrm{~min}$ reaction time at both room temperature and $35^{\circ} \mathrm{C}$ are not significantly better. For the digital image analysis results, the precisions at $30 \mathrm{~min}, 40 \mathrm{~min}$ and $24 \mathrm{~h}$ for the $10 \mu \mathrm{g} \mathrm{L}^{-1}$ solution are significantly smaller than the value for $20 \mathrm{~min}$; however, for the $50 \mu \mathrm{g} \mathrm{L}^{-1}$ solution, only the precision at $40 \mathrm{~min}$ is significantly better than that for $20 \mathrm{~min}$. We conclude that the use of digital image analysis coupled with increased reaction time will give rise to significantly improved precision at these two critical concentration values and thus would decrease the number of false positive and false negative outcomes compared with the numbers obtained by visual comparison of the developed test strips. 


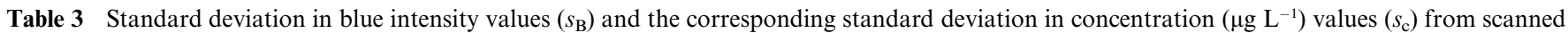
strips run for $20,30,40 \mathrm{~min}, 24 \mathrm{~h}$ at $20^{\circ} \mathrm{C}$ and for $20 \mathrm{~min}$ at $35^{\circ} \mathrm{C}$ ( $n=5$ for all experiments)

\begin{tabular}{|c|c|c|c|c|c|c|c|c|c|c|}
\hline $\begin{array}{l}\text { Concentration of } \\
\text { arsenic }\left(\mu \mathrm{L} \mathrm{L}^{-1}\right)\end{array}$ & $20 \min s_{\mathrm{B}}$ & $\begin{array}{l}20 \min _{\left(\mu \mathrm{L}^{-1}\right)} \\
(\mu \mathrm{g}\end{array}$ & $30 \min s_{\mathrm{B}}$ & $\begin{array}{l}30 \min _{\mathrm{c}} \\
\left(\mu \mathrm{g} \mathrm{L}^{-1}\right)\end{array}$ & $40 \min s_{\mathrm{B}}$ & $\begin{array}{l}40 \min s_{\mathrm{c}} \\
\left(\mu \mathrm{g} \mathrm{L}^{-1}\right)\end{array}$ & $24 \mathrm{~h} s_{\mathrm{B}}$ & $\begin{array}{l}24 \mathrm{~h} s_{\mathrm{c}} \\
\left(\mu \mathrm{g} \mathrm{L}^{-1}\right)\end{array}$ & $35^{\circ} \mathrm{C} s_{\mathrm{B}}$ & $\begin{array}{l}35^{\circ} \mathrm{C} \\
s_{\mathrm{c}}\left(\mu \mathrm{g} \mathrm{L}^{-1}\right)\end{array}$ \\
\hline 10 & 3.8 & 6.2 & 12.1 & 1.5 & 6.3 & 2.3 & 16.0 & 2.4 & 12.9 & 7.2 \\
\hline 50 & 13.3 & 15 & 9.8 & 6.5 & 4 & 4.0 & 4.9 & 6.6 & 18.3 & 9.4 \\
\hline 100 & 18.6 & 45 & 13.5 & 43 & 13 & 35 & 33.5 & $\sim^{a}$ & 19.1 & 55 \\
\hline 250 & 11.4 & 72 & 18.5 & 230 & 11.8 & 158 & 11.5 & 52 & 16.2 & 30.5 \\
\hline 500 & 20.2 & 155 & 24.9 & $-^{a}$ & 8.6 & 219 & 2.4 & 40 & 4.24 & 22.1 \\
\hline
\end{tabular}

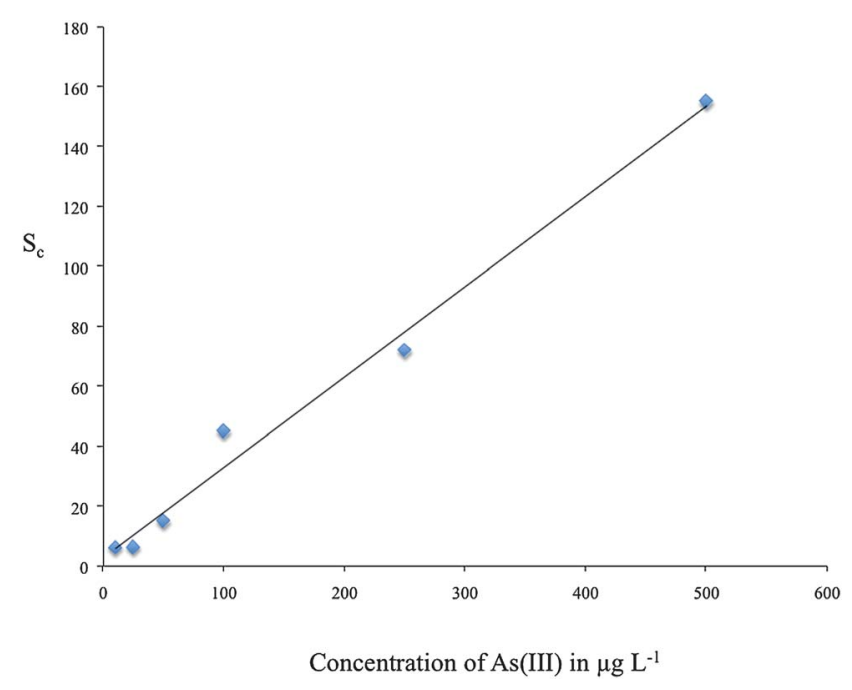

Fig. 3 Plot of $S_{\text {c }}$ versus concentration of arsenic in $\mu \mathrm{g} \mathrm{L}^{-1}$ for $20 \mathrm{~min}$ reaction time. The line is the best fit by the method of least squares.

Table 4 Standard deviations in concentration units at 10 and $50 \mu \mathrm{g} \mathrm{L}^{-1}$, $s_{10}$ and $s_{50}$, at various times at room temperature and for $20 \mathrm{~min}$ at $35^{\circ} \mathrm{C}$

\begin{tabular}{llcllll}
\hline & $\begin{array}{l}\text { Kinniburgh and }_{\text {Kosmus model }}^{6} \\
\text { Kon }\end{array}$ & $20 \mathrm{~min}$ & $30 \mathrm{~min}$ & $40 \mathrm{~min}$ & $24 \mathrm{~h}$ & $35^{\circ} \mathrm{C}$ \\
\hline$s_{10}$ & 10.3 & 6.2 & $1.5^{a b}$ & $2.3^{a b}$ & $2.4^{a b}$ & 7.2 \\
$s_{50}$ & 22 & 15 & $6.5^{a}$ & $4.0^{a b}$ & $6.6^{a}$ & 9.4
\end{tabular}

${ }^{a}$ Value significantly smaller than that of the Kinniburgh and Kosmus model. ${ }^{b}$ Value significantly smaller than that for $20 \mathrm{~min}$ reaction time.

\section{Accuracy}

The effects of reaction time and temperature are clearly quite marked, but with the possible exception of the results for the colours developed after $24 \mathrm{~h}$, the colours for all concentrations are "lighter" than those printed in the chart supplied by the manufacturer. Visual observation under normal laboratory lighting conditions (a mixture of diffuse daylight and fluorescent strips) supports this general observation. This raises the issue about the accuracy of the test as performed under normal laboratory conditions according to the manufacturer's instructions $\left(20^{\circ} \mathrm{C}\right.$ for $20 \mathrm{~min}$ ). One possible reason for the discrepancy is that the light source in the scanner (a cold cathode fluorescent tube) has a spectral output that produces responses to the printed chart and an "exposed" test-strip that are different from those that would be observed in daylight (assuming that this is what the manufacturer has in mind). We have not, as yet, investigated the effect of the light source on the image characteristics. However, as the responses for exposure for $24 \mathrm{~h}$ and at $35{ }^{\circ} \mathrm{C}$ are much closer to those of the printed chart, it seems unlikely that the light source is a major source of inaccuracy, even though the chemical species responsible for the colour on an exposed strip and on the printed chart are quite different.

\section{Conclusions}

Compared with visual comparison of the exposed strips with the printed calibration chart, analysis by the evaluation of the digital images created with a flat-bed scanner is more precise and thus leads to a methods that will give rise to fewer false positive or false negative values at the critical decision values of 10 and $50 \mu \mathrm{g} \mathrm{L}^{-1}$. The intensities of the colored spots are functions of reaction time and temperature, both of which affect the accuracy of the kit. The results indicate that under the recommended operating conditions, the reactions responsible for the colour formation are not complete. The experiments reported here do not allow a distinction between the processes of (a) generation and evolution of arsine into the head-space and (b) reaction of arsine with the mercuric bromide in the strip. Experiments currently in progress suggest that both processes are slow, but that the evolution is slower that the reaction between arsine and the mercuric bromide. Preliminary results also indicate that the rate of evolution is strongly dependent on the nature of the agitation of the contents of the reaction vessel. We suggest that when reporting on the performance of such test kits, information is provided about the reaction temperature, exposure time of the strips, and the lighting conditions under which comparisons were made. The quite large deviations from the colours in the printed chart that were observed are cause for concern, and we suggest that a better strategy would be to calibrate at the time of analysis by measuring solutions of known concentrations. This, of course, raises practical difficulties for genuine field deployment of the test and requires operators to have access to the supplies and facilities necessary to prepare the appropriate standard solutions. There is also the issue of the instability of the colours once formed (especially if exposed to light) and so replacing the printed chart with a set of exposed strips is not a viable strategy. We suggest that this calibration strategy be combined with digital image analysis and applied to every batch of reagents (the Hach kits are supplied with enough reagents to perform 100 tests). We 
also propose that the scanner could be replaced by a digital camera. Calibration in duplicate based on 5 standards, chosen to match the likely range of concentrations encountered, would consume $10 \%$ of the analytical capability of the batch or reagents raising the cost (at the time of writing) by just under $\$ 0.04$ per test. If the target samples are likely to contain concentrations around the WHO critical value of $10 \mu \mathrm{g} \mathrm{L}^{-1}$, we suggest that the 24 hour version of the test be adopted. We realize that this probably means taking samples back to a laboratory of some sort, and almost certainly requires that more reaction vessels be available, as well as limiting the numbers of samples that can be processed in any given time. We expect that the 24 hour version of the test would have a lower detection limit, though it will be necessary to establish the mathematical relationship between colour intensity and concentration.

\section{Acknowledgements}

The material is based upon work supported by the National Science Foundation under grant number CHE-0725257.

\section{References}

1 B. K. Mandal and K. T. Suzuki, Talanta, 2002, 58, 201.

2 D. K. Nordstrom, Science, 2002, 296, 2143.

3 R. W. Sambu, Toxicol. Ind. Health, 2008, 27, 217.

4 A. H. Smith, E. O. Lingas and M. Rahman, Bull. W. H. O., 2000, 78, 1093.

5 B. K. Caldwell, J. C. Caldwell, S. N. Mitra and W. Smith, Soc. Sci. Med., 2003, 56, 2089.

6 D. G. Kinniburgh and W. Kosmus, Talanta, 2002, 58, 165.

7 D. Melamed, Anal. Chim. Acta, 2005, 532, 1-13.

8 M. F. Hossain, Agric. Ecosyst. Environ., 2006, 113, 1.
9 M. M. Rahman, D. Mukherjee, M. K. Sengupta, U. K. Chowdhury, D. C. Lodh, S. Roy, M. Selim, Q. Quamruzzaman, A. H. Milton, S. M. Shahidullah, M. T. Rahman and D. Chakraborti, Environ. Sci. Technol., 2002, 36, 5385.

10 A. Van Geen, Z. Cheng, A. A. Seddique, M. A. Hoque, A. Gelman, J. H. Graziano, H. Ahsan, F. Parvez and K. M. Ahmed, Environ. Sci. Technol., 2005, 39, 299-303.

11 C. M. Steinmaus, C. M. George, D. A. Kalman and A. H. Smith, Environ. Sci. Technol., 2006, 40, 3362.

12 J. F. Tyson, Development of Measurement Technologies for LowCost, Reliable, Rapid, On-Site Determination of Arsenic Compounds in Water, in Contamination of Groundwater: Mechanism, Analysis, and Remediation, ed. S. Ahuja, Wiley, New York, 2008, pp. 147-178.

13 Environmental Technology Verification Program, A review of commercial field test kits for the measurement of arsenic in drinking water 2010, http://www.epa.gov/etv/, 2010, accessed 01 August 2011.

14 M. Thompson and R. J. Howarth, Analyst, 1976, 100, 609.

15 S. G. Reeves, M. A. Roberts, S. T. A. Siebert and R. A. Durst, Anal. Lett., 1993, 26, 1461.

16 S. T. A. Siebert, S. G. Reeves and R. A. Durst, Anal. Chim. Acta, 1993, 282, 297.

17 S. V. Bannur, S. V. Kulgod, S. S. Metkar, S. K. Mahajan and J. K. Sainsi, Anal. Biochem., 1999, 267, 382.

18 M. E. Johnson, J. Chem. Educ., 2000, 77, 368.

19 R. V. Abrazheev, A. D. Zorin, N. P. Savinova and Yu. I. Sannikova, J. Anal. Chem., 2002, 57, 280.

20 M. Kompany-Zareh, M. Mansourian and F. Ravaee, Anal. Chim. Acta, 2002, 471, 97.

21 S. Paciornik, A. V. Yallouz, R. C. Campos and D. Gannerman, J. Braz. Chem. Soc., 2006, 17, 156.

22 R. D. Sharma, S. Joshi and S. Amlathe, Anal. Methods, 2011, 3, 452 .

23 K. R. Mathews, J. D. Landmark and D. F. Stickle, J. Chem. Educ., 2004, 81, 701.

24 http://pubs.acs.org/doi/suppl/10.1021/ed081p.702, accessed 22 September 2011.

25 M. Salman, M. Athar, W. -uz-Zaman, U. Shafique, J. Anwar, R. Rehman, S. Ameer and M. Azeem, Anal. Methods, 2012, 4, 242. 